\title{
Blood volume measurement with indocyanine green pulse spectrophotometry: dose and site of dye administration
}

\author{
Menno R. Germans • Philip C. de Witt Hamer • \\ Leonard J. van Boven • Koos A. H. Zwinderman • \\ Gerrit J. Bouma
}

Received: 12 May 2009/Accepted: 14 August 2009/Published online: 4 September 2009

(C) The Author(s) 2009. This article is published with open access at Springerlink.com

\begin{abstract}
Background (1) To determine the optimal administration site and dose of indocyanine green (ICG) for blood volume measurement using pulse spectrophotometry, (2) to assess the variation in repeated blood volume measurements for patients after subarachnoid hemorrhage and (3) to evaluate the safety and efficacy of this technique in patients who were treated for an intracranial aneurysm.

Methods Four repeated measurements of blood volume (BV) were performed in random order of bolus dose (10 $\mathrm{mg}$ or $25 \mathrm{mg} \mathrm{ICG)} \mathrm{and} \mathrm{venous} \mathrm{administration} \mathrm{site} \mathrm{(peripheral} \mathrm{or}$ central) in eight patients admitted for treatment of an intracranial aneurysm. Another five patients with subarachnoid hemorrhage underwent three repeated BV measurements with $25 \mathrm{mg}$ ICG at the same administration site to assess the coefficient of variation.

Findings The mean $\pm \mathrm{SD}$ in $\mathrm{BV}$ was $4.38 \pm 0.881(\mathrm{n}=25)$ and $4.69 \pm 1.11 \mathrm{l}(\mathrm{n}=26)$ for $10 \mathrm{mg}$ and $25 \mathrm{mg}$ ICG, respectively. The mean $\pm \mathrm{SD}$ in $\mathrm{BV}$ was $4.59 \pm 1.151(\mathrm{n}=$ 26) and $4.48 \pm 0.861(\mathrm{n}=25)$ for central and peripheral administration, respectively. No significant difference was
\end{abstract}

M. R. Germans $(\square)$ • P. C. de Witt Hamer · G. J. Bouma Department of Neurosurgery, Amsterdam, The Netherlands, Neurosurgical Center Amsterdam,

Room H6-150, P.O. Box 22660, 1100 DD Amsterdam,

The Netherlands

e-mail: M.R.Germans@amc.uva.nl

L. J. van Boven

Department of Radiology, Academic Medical Center,

Amsterdam, The Netherlands

\section{K. A. H. Zwinderman}

Department of Clinical Epidemiology,

Biostatistics and Bioinformatics, Academic Medical Center,

Amsterdam, The Netherlands found. The coefficient of variance of BV measurement with $25 \mathrm{mg}$ of ICG was $7.5 \%$ (95\% CI: $3-12 \%$ ).

Conclusions There is no significant difference between intravenous administration of either 10 or $25 \mathrm{mg} \mathrm{ICG}$, and this can be injected through either a peripheral or central venous catheter. The $7.5 \%$ coefficient of variation in $\mathrm{BV}$ measurements determines the detectable differences using ICG pulse spectrophotometry.

Keywords Indocyanine green $\cdot$ Blood volume determination $\cdot$ Spectrophotometry Pulse densitometry Triple H · Subarachnoid hemorrhage

\section{Introduction}

The majority of patients with an aneurysmal subarachnoid hemorrhage (SAH) have a poor prognosis, with mortality rates as high as $45 \%$ and significant morbidity among the survivors [1]. The major causes of morbidity are the initial bleed, a rebleed, hydrocephalus and delayed ischemic neurological deficits (DIND). Delayed ischemic neurological deficits (DIND) are presumably related to cerebral vasospasm and have a large impact on outcome after SAH $[3,17$, 18]. This ischemic complication occurs in $17-40 \%$ and carries a risk of $10-20 \%$ of permanent disability or death $[12,18]$. A hallmark of current therapy includes induction of hypervolemia, hemodilution and hypertension (triple- $\mathrm{H}$ therapy) aiming to restore cerebral perfusion [12]. This aggressive treatment is controversial, however, and its efficacy has not been proven in prospective studies. Additionally, it has considerable morbidity, such as pulmonary edema and cardiac failure $[4,12]$. In a recent study performed by Hoff et al. [7], no association between daily or cumulative fluid balance and actual circulating blood volume 
was seen. So, to increase therapeutic efficacy of triple-H therapy and decrease morbidity, monitoring of circulating blood volume (BV) prior to and during treatment for symptomatic cerebral vasospasm may be of value $[11,16]$.

Blood volume can be determined using dye dilution $[5,9]$. Determination of $\mathrm{BV}$ using indocyanine green (ICG) administration and detection with pulse spectrophotometry has several advantages. It can be performed in several minutes at the bedside $[5,10,15]$, allowing multiple measurements on a daily basis, limited only by the short washout time of the ICG dye [2]. This technique has been validated in healthy volunteers and is comparable in accuracy to the gold standard isotopic dilution techniques $[6,8]$. Furthermore, ICG pulse spectrophotometry has been safely applied in critically ill patients in the ICU and perioperatively $[9,13,14]$. In our opinion, this technique could be used to optimize the BV status of patients after SAH. However, for the reliable clinical application of this technique in patients with intracranial pathology, several issues need to be addressed. First, the optimal ICG dose site of venous administration (central vs. peripheral) has to be determined, and second, the variation in repeated BV measurements should be assessed to determine the detectable differences.

The aims of this study were (1) to determine the optimal dose and venous administration site for ICG for measurement of BV, (2) to establish the coefficient of variation for repeated BV measurements with pulse spectrophotometry and (3) to evaluate the safety and efficacy of this technique in patients who were treated for an intracranial aneurysm.

\section{Methods and materials}

\section{Patient population}

Adult patients ( $\geq 18$ years) admitted for treatment of an intracranial aneurysm, with or without recent subarachnoid hemorrhage (confirmed on computer tomography scan or by lumbar puncture positive for xanthochromia), were recruited for this study. All patients had a medical indication for both a central and a peripheral venous catheter and were admitted to the intensive care. Exclusion criteria were iodine allergy or increased baseline liver enzymes (AST $>150 \mathrm{U} / 1$ or ALT $>$ $150 \mathrm{U} / 1$ or $\mathrm{AF}>150 \mathrm{U} / 1$ or $\gamma-\mathrm{GT}>150 \mathrm{U} / \mathrm{l}$ ) because ICG is cleared hepatically. The Medical Ethical Committee of the University of Amsterdam approved the study protocol (02/ 203 no. 02.17.1017), and informed consent was obtained from all participants.

Indocyanine green dilution procedure

The Dye Densitogram $2501 \mathrm{~A} / \mathrm{K}$ analyzer (Nihon Kohden, Tokyo, Japan) was used for registration of the spectrophoto- metrical absorption of ICG and calculation of the BV. After an intravenous bolus of ICG (Infracyanine ${ }^{\circledR}$, S.E.R.B. laboratories pharmaceutiques, Paris, France) in glucose 5\% solution, the decay curve in the peripheral tissue was assessed spectrophotometrically with a light probe attached to the nasal wing for $10 \mathrm{~min}$. Because ICG does not leave the intravascular compartment, the diluting volume for ICG equals the BV. The diluting volume can be calculated from the decay curve by regression [5], and measurements can be repeated after 30 min when all ICG has been cleared by the liver [6]. Further details have been described elsewhere $[8,9]$.

Study protocol

In this study two successive protocols were used. The first was to determine the optimal dose and administration site, followed by the establishment of the coefficient of variation.

In the first protocol, ICG powder was dissolved in glucose $5 \%$ to a concentration of $5 \mathrm{mg} / \mathrm{ml}$, from which either $5 \mathrm{ml}$ (25 mg ICG) or $2 \mathrm{ml}$ (10 $\mathrm{mg}$ ICG) was used as bolus. The bolus was manually infused within $5 \mathrm{~s}$ through either a peripheral or central venous catheter followed by $20 \mathrm{ml}$ or $23 \mathrm{ml}$ glucose $5 \%$ flush, respectively. Using the pulse spectrophotometer probe attached to the nasal wing, dye absorption was instantaneously measured for the following $10 \mathrm{~min}$. All patients received every single combination between dose and administration site with 30-min intervals. The order of combinations was randomized in advance.

The four measurements consisted of two paired observations of $10 \mathrm{mg}$ ICG versus $25 \mathrm{mg}$ ICG and two paired observations of central versus peripheral venous administration site. Patients who were eligible for more than one series of four repeated measurements participated to a maximum of three separated series. Because of the admission of $25 \mathrm{ml}$ of the study agent in four repeated BV measurements reduced by continuous diuresis, the net result in fluid balance was assumed to be constant during the experiment.

To determine the coefficient of variance, three repeated BV measurements using $25 \mathrm{mg}$ ICG through the same venous administration site were performed in a period with 30-min intervals. For this protocol, five different patients with recent SAH were included who had not participated in the first part of this study.

\section{Statistics}

The BV difference between low- and high-dose ICG and between central and peripheral venous administration was hypothesized to be less than 5\%. Assuming a standard deviation of $5 \%$, a power of 0.90 and an $\alpha$-level of 0.05 , a minimum of 23 paired observations were required. A linear mixed model was used to establish differences in BV 
Table 1 Patients characteristics and interval between hemorrhage and BV measurement of first study population (see text)

\begin{tabular}{|c|c|c|c|c|c|c|c|c|c|}
\hline Patient & Gender & $\begin{array}{l}\text { Age } \\
\text { (years) }\end{array}$ & $\begin{array}{l}\text { Weights } \\
(\mathrm{kgs})\end{array}$ & $\begin{array}{l}\text { Hunt and } \\
\text { Hess }\end{array}$ & $\begin{array}{l}\text { Localisation of } \\
\text { aneurysm }\end{array}$ & Treatment & Triple-H & Hyponatriaemia & $\begin{array}{l}\text { Days between SAH } \\
\text { and measurement(s) }\end{array}$ \\
\hline 1 & $\mathrm{~F}$ & 40 & 62.0 & I & $\mathrm{ACM}$ & Clip & Yes & No & 10 \\
\hline 2 & M & 39 & 70.0 & 0 & PICA & Clip & No & No & n.a. \\
\hline 3 & $\mathrm{~F}$ & 41 & 70.0 & II & P.comm. & Clip & No & Yes & 3 \\
\hline 4 & $\mathrm{~F}$ & 42 & 60.0 & III & $\mathrm{ACM}$ & Clip & Yes & No & $\begin{array}{l}\text { (1) } 21 \\
\text { (2) } 22\end{array}$ \\
\hline 5 & $\mathrm{~F}$ & 54 & 79.0 & II & $\mathrm{ACM}$ & Clip & No & No & $\begin{array}{l}\text { (1) } 2 \\
\text { (2) } 3 \\
\text { (3) } 6\end{array}$ \\
\hline 6 & $\mathrm{~F}$ & 66 & 66.0 & III & P.comm. & Clip & No & Yes & $\begin{array}{l}\text { (1) } 15 \\
\text { (2) } 16\end{array}$ \\
\hline 7 & M & 51 & 80.0 & II & Ant.chor. & Clip & Yes & No & $\begin{array}{l}\text { (1) } 7 \\
\text { (2) } 8\end{array}$ \\
\hline 8 & $\mathrm{~F}$ & 38 & 60.0 & II & $\mathrm{ACM}$ & Clip & No & No & 36 \\
\hline
\end{tabular}

ACM: middle cerebral artery; PICA: posterior inferior cerebellar artery; p.comm.: posterior communicating artery; Ant.chor.: anterior choroideal artery

between low and high dose ICG and central and peripheral administration site.

Data analysis was performed using SPSS 16.0.1 (SPSS Inc, Chicago, IL) with the restricted maximum likelihood estimation method using BV as independent variable. ICG dose, administration site and the interaction of these were included as fixed effects, and patient and repeated measurement series identification as random effects.

\section{Results}

The first study population consisted of two males and six females with a mean age of $46.4 \pm 9.8$ years. Seven patients presented with a ruptured aneurysm and one with an incidental aneurysm discovered by computer tomography angiography. Five aneurysms were situated in the anterior circulation, and there were two posterior communicating artery and one posterior inferior cerebellar artery aneurysms; all were treated surgically. Three patients had been treated for DIND with triple-H therapy, and during the repeated BV measurements none of the patients was suspected of having symptomatic cerebral vasospasm, evaluated by neurological examination. Two patients had a mild hyponatriaemia (130 $135 \mathrm{mmol} / \mathrm{l}$ ) during measurements; these had not been previously treated with triple-H therapy (see Table 1).

In 8 patients, $51 \mathrm{BV}$ measurements were done resulting in 25 paired observations. One patient completed three series of the four repeated measurements on separate days, three patients completed two series, and four patients completed one series. An ICG dose of $10 \mathrm{mg}$ and $25 \mathrm{mg}$ was administered in 25 and 26 measurements, respectively. A central venous catheter and a peripheral venous catheter were used for administration in respectively 26 and 25 measurements. One
Fig. 1 Results of blood volume measurements. On x-axis the combination of dose and site administration with means \pm standard deviation: $1=$ central $10 \mathrm{mg}: 4.28 \pm 0.941(\mathrm{n}=13)$; $2=$ central $25 \mathrm{mg}: 4.90 \pm$ $1.281(\mathrm{n}=13) ; 3=$ peripheral $10 \mathrm{mg}: 4.49 \pm 0.841(\mathrm{n}=12)$; 4= peripheral $25 \mathrm{mg}: 4.47 \pm$ $0.911(\mathrm{n}=13)$

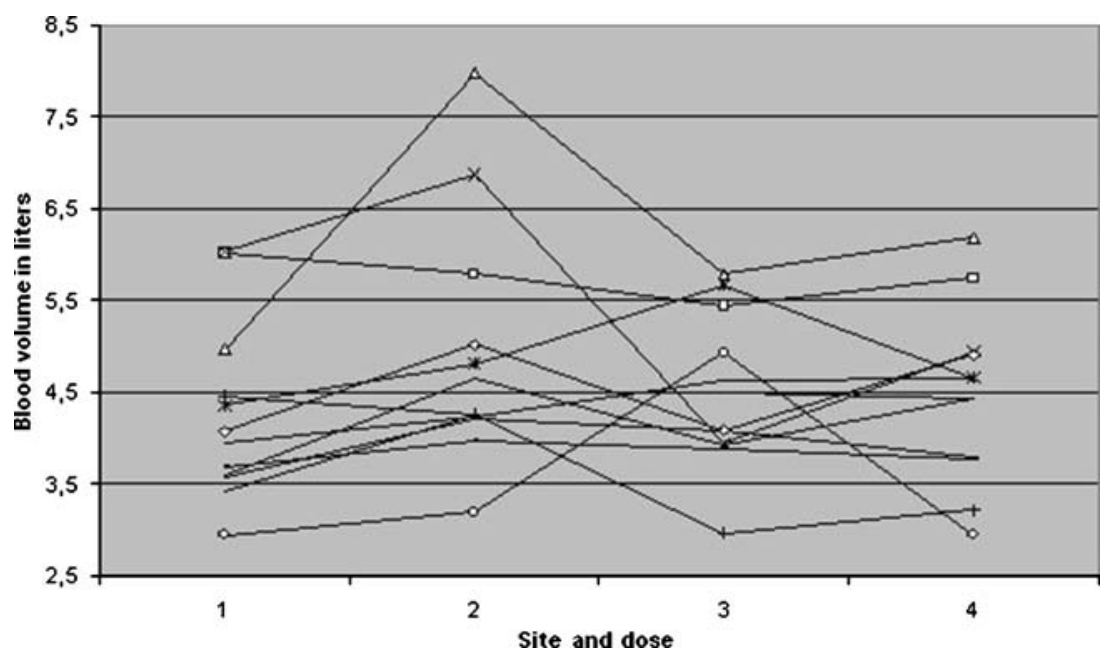


Table 2 Patients characteristics and interval between hemorrhage and BV measurement of second study population (see text)

\begin{tabular}{|c|c|c|c|c|c|c|c|c|}
\hline Patient & Gender & $\begin{array}{l}\text { Age } \\
\text { (years) }\end{array}$ & Hunt and Hess & Localisation & Treatment & Triple-H & Hyponatriaemia & $\begin{array}{l}\text { Days between SAH } \\
\text { and measurements }\end{array}$ \\
\hline $\mathrm{a}$ & $\mathrm{F}$ & 74 & II & P.comm. & Clip & No & No & 11 \\
\hline $\mathrm{b}$ & M & 70 & II & - & - & No & No & 7 \\
\hline $\mathrm{c}$ & M & 61 & I & A.comm. & Coil & No & Yes & 13 \\
\hline d & $\mathrm{F}$ & 69 & III & A.comm. & Clip & Yes & Yes & 22 \\
\hline e & M & 57 & II & Bas.top & Coil & No & No & 6 \\
\hline
\end{tabular}

ACM: middle cerebral artery; PICA: posterior inferior cerebellar artery; p.comm.: posterior communicating artery; a.comm.: anterior communicating artery

measurement of $10 \mathrm{mg}$ ICG bolus through a peripheral venous catheter was not completed, because the patient withdrew from further participation due to pain at the injection site at bolus injection. No other adverse events were observed.

The mean \pm SD of the BV measured by $10 \mathrm{mg}$ and $25 \mathrm{mg}$ ICG was $4.38 \pm 0.881(\mathrm{n}=25)$ and $4.69 \pm 1.111(\mathrm{n}=26)$, respectively. At central and peripheral administration, it was $4.59 \pm 1.151(\mathrm{n}=26)$ and $4.48 \pm 0.861(\mathrm{n}=25)$, respectively (Fig. 1). Using the linear mixed model, we calculated comparable BVs in the two sites of administration (estimate $=0.431, \mathrm{SE}=0.27, \mathrm{p}=0.126$ ), as well in the two different doses (estimate $=0.021, \mathrm{SE}=0.28, \mathrm{p}=0.953$ ). With these results we could not determine a significant difference between the ICG dose nor the administration site.

Subsequently, the coefficient of variance was measured in three repeated BV measurements in five patients, two females and three males with a mean age of $64 \pm 9$ years (patients a to e; see Table 2). Calculated BVs of the three measurements in every patient (a to e) are plotted in Fig. 2. The mean \pm SD of the coefficient of variation for a $25 \mathrm{mg}$ ICG bolus in five patients was $7.5 \% \pm 3.6 \%$ resulting in a $95 \%$ CI between $3 \%$ and $12 \%$.

\section{Discussion}

For determination of BV by ICG pulse spectrophotometry in patients being treated for an intracranial aneurysm, there is no significant difference between a bolus ICG dose of 10 or $25 \mathrm{mg}$ through either a central or peripheral venous catheter. Earlier reports using ICG spectrophotometry in critically ill patients describe varying dosages of ICG between 10 and $25 \mathrm{mg}[5,6,8-11,14,15]$. It seems reasonable to use a standardized ICG bolus dose; in the calculation for variance, we have chosen $25 \mathrm{mg}$ to minimize the error of spilling ICG at injection. As can be concluded from these data, the theoretical delay to mean transit time from peripheral injection does not substantially affect BV measurements in adult patients with average weight. This is in contrast with $\mathrm{He}$ et al. [6], who suggested that the mean transit time depends on the size of the subject. As they drew their conclusion after comparison between adults and children with diverse body surface areas, no conclusive statement can be made concerning this issue.

When evaluating the larger than average BV measurements, no direct relation was seen between previous triple$\mathrm{H}$ therapy or hyponatriemia during the measurement except for one patient. His BV was larger than the standard deviation in three of four measurements, explained by the preceded triple- $\mathrm{H}$ therapy 2 days before. The absence of a relation is in accordance with the conclusion of a recent study of Hoff et al. [7] who "...raises doubt whether fluid management guided by fluid balances is effective...."

During the inclusion period also one patient without a recent SAH was treated. Theoretically, this could confound the results because $\mathrm{BV}$ in a presumed healthy patient could be different as compared with patients with a recent SAH. But in our opinion, measurements in this patient did not influence the results because treatment of this patient was identical to patients with SAH and an uncomplicated course.

With the standard 25-mg dose injected through either venous administration site, the coefficient of variance of BV measurements appears to be $7.5 \%$. Taking the $95 \%$ confidence

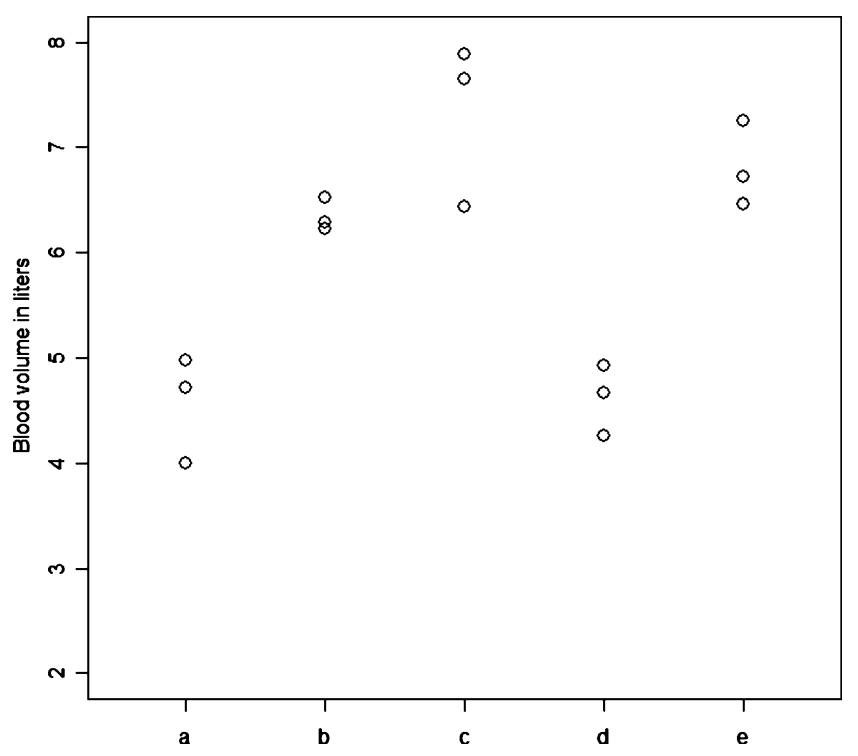

Fig. 2 Repeated blood volume measurements in separate patients (a to e) with $25 \mathrm{mg}$ ICG through the same administration site 
interval into account, it seems reasonable to assume that the mean differences in BV of more than $12 \%$ can be detected significantly using this technique. This percentage is an important baseline parameter for planning further studies.

One study using repeated BV measurements with ICG pulse spectrophotometry in healthy volunteers determined a variation of $3.94 \%$ [6]. The larger variation in our data can be explained by the different measurement circumstances and smaller number of patients. The measurements in our study were performed when the patient was awake, contrary to the patients who were under general anesthesia in the study of $\mathrm{He}$ et al. Apparently, awake patients induce more measurement artifacts, probably by probe movement or changes in cardiac output during the 10-min pulse spectrophotometry or between successive measurements. Nevertheless, our results show that reliable BV measurements can also be performed in awake patients with recent $\mathrm{SAH}$.

Our percentage of coefficient of variance is an important baseline parameter for planning further studies concerning patients with SAH.

Blood volume can be determined in a safe and reproducible manner in patients after SAH using ICG pulse spectrophotometry. Administration of a bolus of either 10 or $25 \mathrm{mg}$ ICG through a central or peripheral catheter results in no significant differences in measured BV. To minimize the error of spilling during injection, we recommend 25-mg boluses for repeated BV measurements. The coefficient of variation of repeated measurements with $25 \mathrm{mg}$ is estimated at $7.5 \%$. These results are considered important premises for planning further clinical studies.

Open Access This article is distributed under the terms of the Creative Commons Attribution Noncommercial License which permits any noncommercial use, distribution, and reproduction in any medium, provided the original author(s) and source are credited.

\section{References}

1. Bederson JB, Connolly ES Jr, Batjer HH, Dacey RG, Dion JE, Diringer MN, Duldner JE Jr, Harbaugh RE, Patel AB, Rosenwasser RH (2009) Guidelines for the management of aneurysmal subarachnoid hemorrhage: a statement for healthcare professionals from a special writing group of the Stroke Council, American Heart Association. Stroke 40(3):994-1025

2. Cherrick GR, SW Stein, Leevy CM, CS Davidson (1960) Indocyanine green: observations on its physical properties, plasma decay, and hepatic extraction. J Clin Invest 39:592-600

3. de Oliveira JG, Beck J, Ulrich C, Rathert J, Raabe A, Seifert V (2007) Comparison between clipping and coiling on the incidence of cerebral vasospasm after aneurysmal subarachnoid hemorrhage: a systematic review and meta-analysis. Neurosurg Rev 30(1):22-30

4. Egge A, Waterloo K, Sjoholm H, Solberg T, Ingebrigtsen T, Romner B (2001) Prophylactic hyperdynamic postoperative fluid therapy after aneurysmal subarachnoid hemorrhage: a clinical, prospective, randomized, controlled study. Neurosurgery 49(3):593-605
5. Haruna M, Kumon K, Yahagi N, Watanabe Y, Ishida Y, Kobayashi N, Aoyagi T (1998) Blood volume measurement at the bedside using ICG pulse spectrophotometry. Anesthesiology 89(6):1322-1328

6. He YL, Tanigami H, Ueyama H, Mashimo T, Yoshiya I (1998) Measurement of blood volume using indocyanine green measured with pulse-spectrophotometry: its reproducibility and reliability. Crit Care Med 26(8):1446-1451

7. Hoff RG, van Dijk GW, Algra A, Kalkman CJ, Rinkel GJ (2008) Fluid balance and blood volume measurement after aneurysmal subarachnoid hemorrhage. Neurocrit Care 8(3):391-397

8. Iijima T, Iwao Y, Sankawa H (1998) Circulating blood volume measured by pulse dye-densitometry: comparison with (131)IHSA analysis. Anesthesiology 89(6):1329-1335

9. Ishihara H, Iwakawa T, Hasegawa T, Muraoka M, Tsubo T, Matsuki A (1999) Does indocyanine green accurately measure plasma volume independently of its disappearance rate from plasma in critically ill patients? Intensive Care Med 25(11):1252-1258

10. Kasuya H, Onda H, Yoneyama T, Sasaki T, Hori T (2003) Bedside monitoring of circulating blood volume after subarachnoid hemorrhage. Stroke 34(4):956-960

11. Nakagawa A, Su CC, Sato K, Shirane R (2002) Evaluation of changes in circulating blood volume during acute and very acute stages of subarachnoid hemorrhage: implications for the management of hypovolemia. J Neurosurg 97(2):268-271

12. Naval NS, Stevens RD, Mirski MA, Bhardwaj A (2006) Controversies in the management of aneurysmal subarachnoid hemorrhage. Crit Care Med 34(2):511-524

13. Sakka SG, Reinhart K, Meier-Hellmann A (2000) Comparison of invasive and noninvasive measurements of indocyanine green plasma disappearance rate in critically ill patients with mechanical ventilation and stable hemodynamics. Intensive Care Med 26(10):1553-1556

14. Sakka SG, Reinhart K, Wegscheider K, Meier-Hellmann A (2002) Comparison of cardiac output and circulatory blood volumes by transpulmonary thermo-dye dilution and transcutaneous indocyanine green measurement in critically ill patients. Chest 121(2):559-565

15. Sato K, Karibe H, Yoshimoto T (1999) Circulating blood volume in patients with subarachnoid haemorrhage. Acta Neurochir (Wien ) 141(10):1069-1073

16. Solomon RA, Post KD, McMurtry JG III (1984) Depression of circulating blood volume in patients after subarachnoid hemorrhage: implications for the management of symptomatic vasospasm. Neurosurgery 15(3):354-361

17. Stein SC, Levine JM, Nagpal S, LeRoux PD (2006) Vasospasm as the sole cause of cerebral ischemia: how strong is the evidence? Neurosurg Focus 21(3):E2

18. Weyer GW, Nolan CP, Macdonald RL (2006) Evidence-based cerebral vasospasm management. Neurosurg Focus 21(3):E8

\section{Comment}

The authors provide a means for easy and consistent measurement of BV in patients with SAH. The further perspective is to validate in due time the use of triple-H therapy in these patients by having a measurement tool to know whether "volume" interventions really do increase circulating blood volume. The authors have adopted an existing technique and have standardized this in a sensible matter: one place for infusion of dye, one concentration for all. The results prove rather adequately that this is possible. The neurosurgical community will look forward to further studies with the use of this technique that may prove or disprove that "single-," "double-" or "triple"-H therapy has a physiological basis and effect in the treatment of secondary ischemia after SAH!

\section{J.J.A. Mooij}

Groningen, The Netherlands 